\title{
LETTER \\ Robot Exploration in a Dynamic Environment Using Hexagonal Grid Coverage
}

\author{
Kihong $\mathrm{KIM}^{\dagger}$, Nonmember and SeongOun HWANG ${ }^{\dagger \mathrm{a})}$, Member
}

SUMMARY Robot covering problem has gained attention as having the most promising applications in our real life. Previous spanning tree coverage algorithm addressed this problem well in a static environment, but not in a dynamic one. In this paper, we present and analyze our algorithm workable in a dynamic environment with less shadow areas.

key words: robotics, spanning tree coverage, exploration, hexagonal grid coverage

\section{Introduction}

The robot covering problem can be formulated as follows. Given a continuous work area, a robot moves along a path such that every area in the work area is covered along the path. Robot covering is one of very promising applications such as cleaning, lawn mowing, harvesting, search and rescue, and mine detection in our life.

Traditional coverage algorithms used by cleaning robots are based on random moving ([1], [2]). However, they have a shortcoming that they take a long time to complete their mission. To deal with this problem, a lot of researches have been made. One representative approach is based on spanning tree coverage (STC), where a robot decomposes a work area into complete square-shaped cells, makes a spanning tree, and covers the entire work area [3]. STC optimally solves the single-robot coverage problem. STC algorithms have also developed into MSTC (multirobot spanning tree coverage) ([4], [5]) and MFC (multirobot forest coverage) algorithms [6]. Existing STC algorithms have focused on two issues: full (a.k.a complete) and non-repetitive coverage. However, those approaches have the following disadvantages:

First, those may not be workable in a specific dynamic environment, where obstacles are unexpectedly added to the work area (for example, in the open air, stones or trees come down and block off the returning path) after a spanning tree was established (See Fig. 1 and Fig. 2).

Second, STC algorithms generate a large portion of shadow areas from the given work area. It is because in STC, each cell of size 2D is partitioned into four sub-cells of size D (See Fig. 3), and the areas denoted by X can be fallen into shadow area even though they are large enough to be passed by a robot (See Fig. 4).

Third, all online versions of those approaches require

Manuscript received June 27, 2013.

Manuscript revised September 2, 2013.

${ }^{\dagger}$ The authors are with Hongik University, Rep. of Korea.

a) E-mail: sohwang@hongik.ac.kr (Corresponding author)

DOI: 10.1587/transinf.E96.D.2877



Fig. 1 A spanning tree was established before an obstacle occurred.

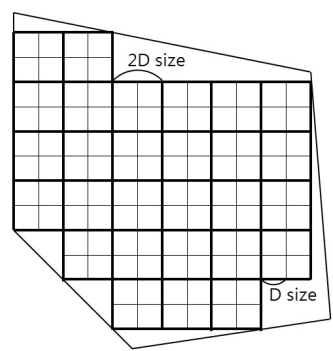

Fig. 3 A work area is divided into 2D-sized cells.



Fig. 2 An obstacle blocked off the returning path of the spanning tree.

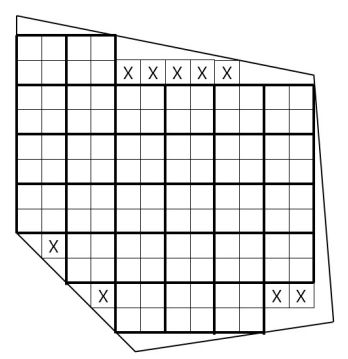

Fig. $4 \mathrm{X}$ shows shadow areas recursive calls whose number grows in linear with the total number of cells within the work area. This is very inappropriate in a mobile robot environment where a robot agent has a very limited memory space but the coverage area is very broad, particularly in outdoor environments.

Therefore, we need a full coverage algorithm workable in a dynamic environment which guarantees non-repetitive or minimal repetitive coverage. In this paper, we present and analyze the algorithm called HGC (hexagonal grid coverage).

\section{The HGC Algorithm}

In case of STC algorithms, there are off-line and on-line versions. The off-line version starts coverage with perfect apriori knowledge of its environment. In contrast, the on-line version starts coverage with partial knowledge of its environment. The knowledge is gradually incremented while covering the work area. HGC algorithm is basically an online version, but also works in off-line way.

In this paper, we make some assumptions as follows: 
Table 1 HGC Algorithm.

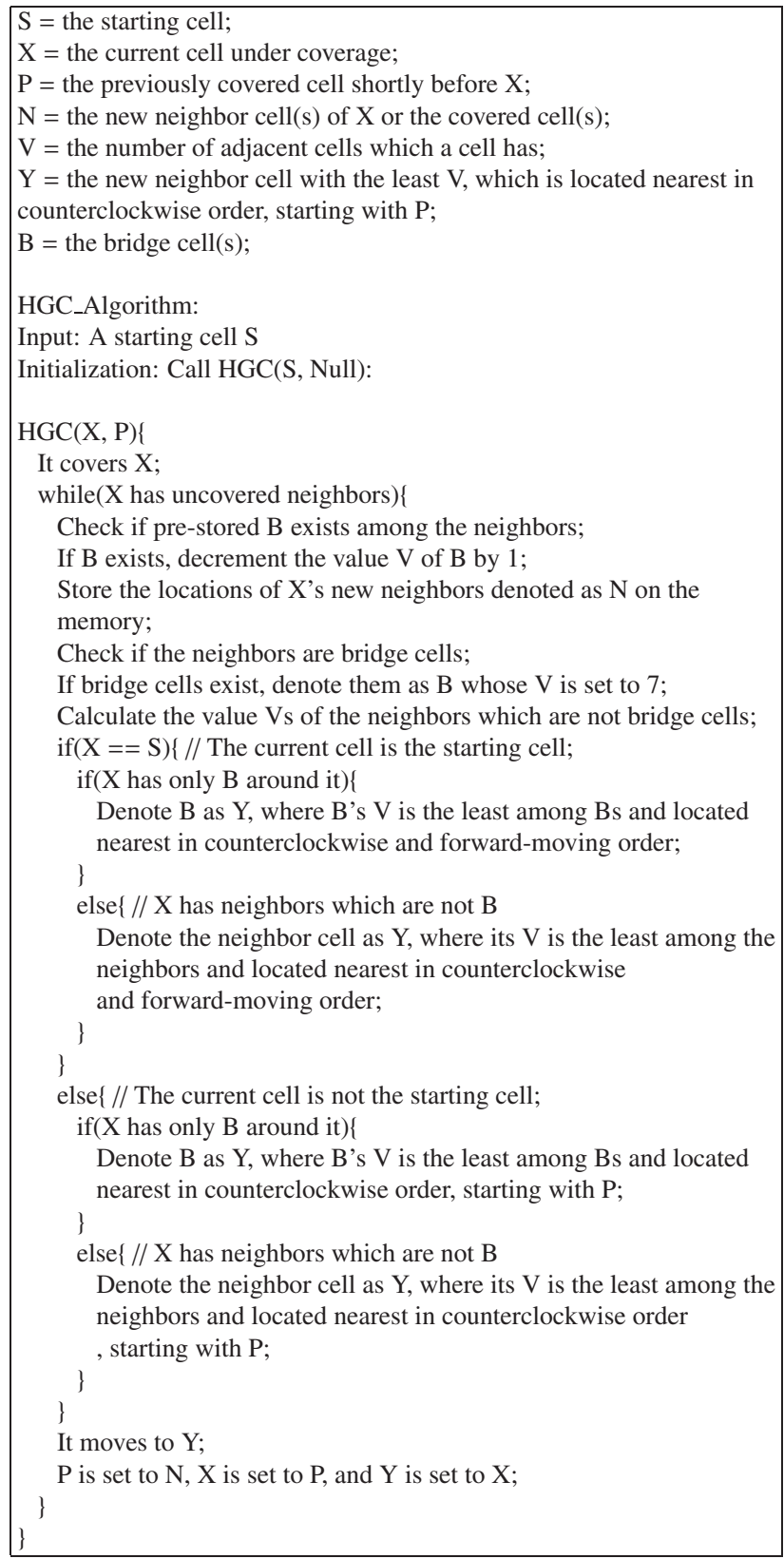

First, we assume that a robot has a tool which is used both to cover the work area and to find obstacles along the path. The tool is assumed to have a size of $\mathrm{D} \mathrm{m}^{2}$ for the convenience of comparison with STC. Second, the shape of tool is hexagonal [7]. This is why we name our algorithm HGC. Third, the robot moves the tool only in directions orthogonal to the tool's six sides while detecting and evading obstacles. Fourth, the robot stores the positions of neighbor cells which are adjacent to the covered cells.

The algorithm starts by setting up parameters as shown in Table 1. After setting up the parameters, the robot moves to the target area and start its coverage. While covering, the robot stores their locations on its memory.

We now explain how the algorithm works in the follow-

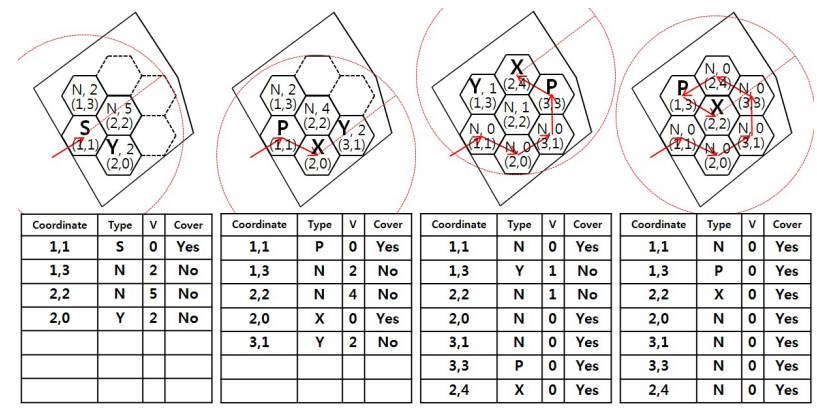

Fig.5 HGC algorithm works in a simple work area with no repetition involved.

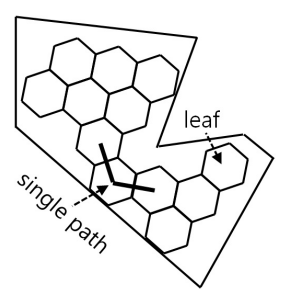

Fig. 6 Leaf cell and single path.

ing simple work area where there is no repetition involved.

In Fig. 5, the robot starts its coverage from the point $\mathrm{S}$. The coordinates of $\mathrm{S}$ are $(1,1)$. At the point $\mathrm{S}$, the robot scans for the neighbor cells $(1,3),(2,2),(2,0)$ and records both the coordinates and $\mathrm{V}$ values of those cells. They are marked as ' $N$ '. As in the first graph of Fig. 5, the cell at $(2,0)$ becomes $\mathrm{Y}$ because its $\mathrm{V}$ value is the least among the three neighbor cells and its location is the nearest to $\mathrm{S}$ in counterclockwise order. The robot moves to the position of $(2,0)$. After the robot covered the cell at $(2,0)$, the cell's type is changed from ' $\mathrm{Y}$ ' to ' $\mathrm{X}$ '. The robot restarts scanning for a new Y. As in the fourth graph of Fig. 5, the robot finishes its coverage. Figure 6 gives an example of work area where both a leaf cell (i.e., a cell with only a single neighbor cell) and a single path exist. However, we only consider a work area where (1) a leaf cell does not exist, and (2) a single path does not exist before the robot starts its coverage. It is because if the work area satisfies one of the above two properties, then repetition of coverage is inevitable. However, while the robot covers the work area, single paths can be ensued. These type of single paths can be handled by our algorithm.

To address the coverage repetition problem accordingly, we introduce the concept of bridge cell in the algorithm. A bridge cell is a cell whose deletion increases the number of connected work areas. A bridge cell is the same as local cut node explained in [3]. In the algorithm, bridge cells are marked as 'B's and their $\mathrm{V}$ values are set to 7 . The algorithm determines a bridge cell when it is located as in Fig. 7, where an unmarked cell is either ' $\mathrm{Y}$ ' or uncovered 'N'.

In the following, we explain how the HGC algorithm works in the same work setting (same area pattern and size) 

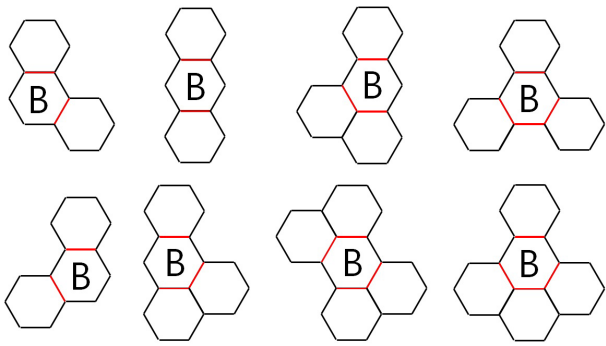

Fig. 7 Bridge cells.

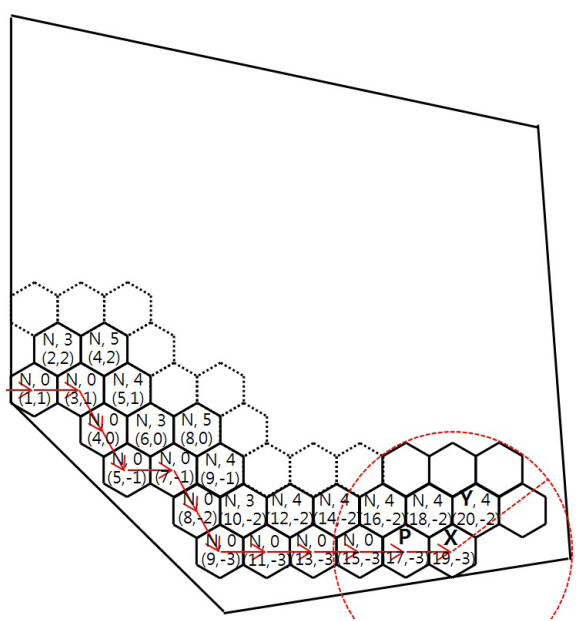

Fig. 8 Coverage made before an obstacle happens.

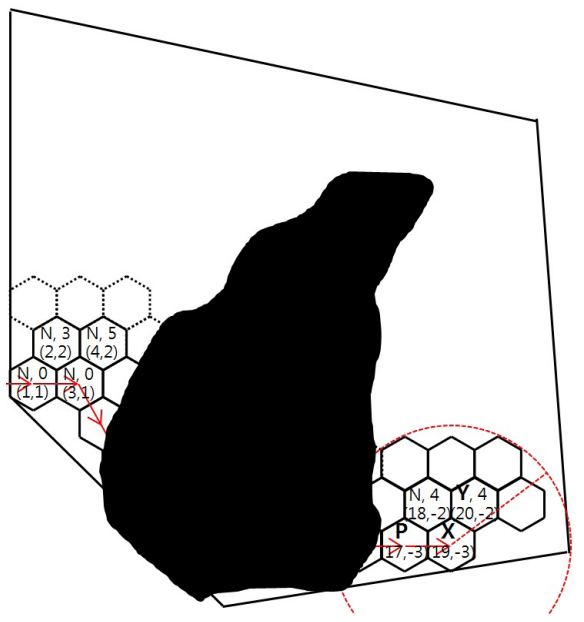

Fig. 9 An obstacle happens.

with obstacles added. We assume that the robot currently covers $(19,-3)$ as in Fig. 8. At that time, an obstacle happened as in Fig. 9. Figure 10 shows that the robot covered up to $(21,5)$. The robot has three new neighbors, whose coordinates are $(20,4),(19,5)$ and $(20,6)$. Among them, $(19,5)$ and $(20,6)$ become bridge cells. The cell at $(20,4)$ becomes $\mathrm{Y}$, which will be covered by the robot at subsequent stage. As in Fig. 11, the robot continues its covering until it reaches $(18,-2)$. Note that there is no Y, but only Bs exist around the



Fig. 10 Identification of bridge cells.

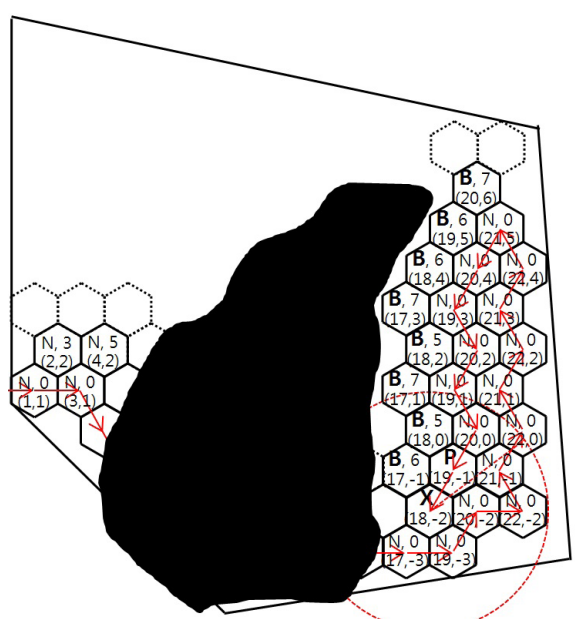

Fig. 11 A single path is formed by bridge cells.

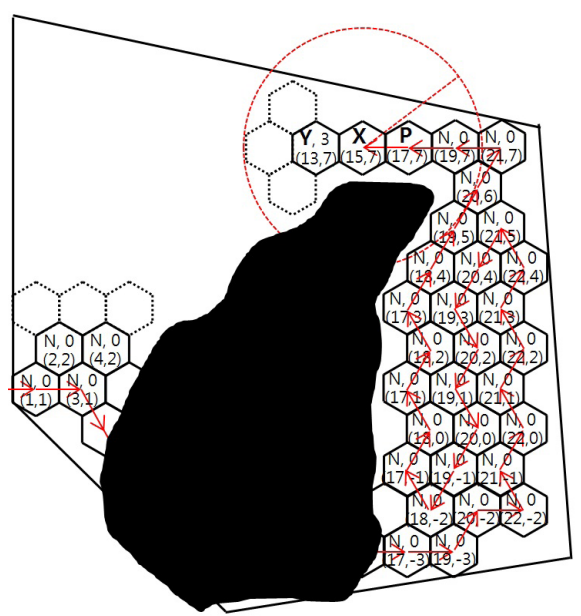

Fig. 12 The robot moves to the other part via the formed single path.

robot. A number of Bs form a single path, which the robot uses to cover the remaining work area as shown in Fig. 12. As in Fig. 13, the robot finally completes its coverage. 




Fig. 13 Coverage is completed.

\section{Algorithm Analysis}

Theorem 1 (Complete coverage): The HGC algorithm completely covers every cell which is accessible from the starting cell S.

Proof: The covering robot divides the work area into a set of cells and stores their locations. The work area consists of hexagonal cells which are identical in shape and size. Each cell has its adjacent cells, whose number ranges at least two through six (a leaf cell which has a single adjacent cell is excluded in the paper). Therefore, the component of the work area grid accessible from $\mathrm{S}$ is a connected graph. The robot can move from the current cell to one of its adjacent cells. There is no gap among cells. Therefore, the robot can cover all cells accessible from $S$.

Theorem 2 (Non-repetitive coverage): The HGC algorithm does not repeatedly cover any point in the work area grid.

Proof: To prevent repetitive coverage, we introduced the concept of bridge cell. Bridge cell is only one path which connects a cell with its adjacent cells. The robot usually probes normal cells at first. The robot continues probing in one direction, and then finally reaches a deadend point. If there is no normal cell, then the robot checks if there is any bridge cell around it. A number of bridge cells form an exit way which allows the robot to get out of the deadend point. In addition, the robot stores the coordinates of the covered area on its memory. Therefore, the robot can cover any work area point without any repetition.

\section{Comparison with STC}

Figure 14 shows cell parameters for comparison of HGC with STC.

Figure 15 shows that the number of cells covered by HGC is greater than those by STC. It means that the size of

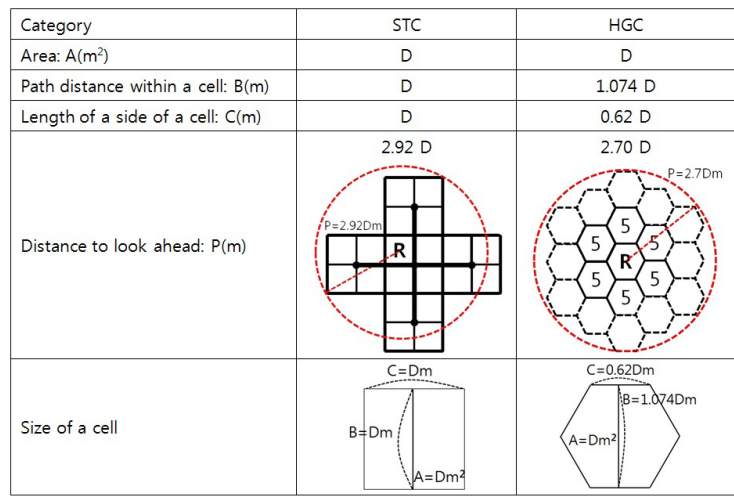

Fig. 14 Parameter setting.



Fig. 15 Comparison between HGC and STC.

shadow areas which the robot cannot cover is decreased in our algorithm. However, the coverage distance is increased a little bit. From simulations in various work areas, we also found that STC is suitable for square-shaped work area, but HGC for rectangle-shaped one. Work areas in real environment are closer to rectangle-shaped areas with a number of curves.

\section{Future Work}

Our future work is to address repetitive coverage in more complex work areas, which are not addressed in the current HGC algorithm and classified into two cases: The first case is, either leaf cells exist or single paths exist. The second case is, the work area gets more complex due to obstacles which happened after the robot starts its coverage. For example, a leaf cell can be generated due to obstacles happening in the way.

\section{Acknowledgements}

This work was supported by 2013 Hongik University Research Fund.

\section{References}

[1] J. Jones, "Robots at the tipping point: The road to the iRobot roomba," IEEE Robotics and Automation Magazine, vol.13, no.1, pp.76-78, 2006.

[2] H. Choset, "Coverage for robotics - a survey of recent results," Annals of Mathematics and Artificial Intelligence, vol.31, no.1-4, pp.113126, 2001. 
[3] Y. Gabriely and E. Rimon, "Spanning-tree based coverage of continuous areas by a mobile robot," Annals of Mathematics and Artificial Intelligence, vol.31, no.1-4, pp.77-98, 2001.

[4] N. Hazon and G.A. Kaminka, "Redundancy, efficiency and robustness in multi-robot coverage," IEEE International Conference on Robotics and Automation, pp.735-741, 2005.

[5] N. Agmon, N. Hazon, and G.A. Kaminka, "Constructing spanning trees for efficient multi-robot coverage," IEEE International Confer- ence on Robotics and Automation, pp.1698-1703, 2006.

[6] X. Zheng, S. Koenig, D. Kempe, and S. Jain, "Multi-robot forest coverage for weighted and unweighted terrain," IEEE Trans. Robotics, vol.26, no.6, pp.1018-1031, 2010.

[7] H.J. Quijano, "Improving cooperative robot exploration using an hexagonal world representation," IEEE Fourth Congress of Electronics, Robotics and Automotive Mechanics, pp.450-455, 2007. 